\title{
Psicopatía, adicciones, violencia interpersonal y conducta antisocial, una relación mixta
}

\section{Psychopathy, Addictions, Interpersonal Violence and Antisocial Behavior, a mixed relationship}

\author{
GERARdo FLóreZ* ** \\ *Unidad de Conductas Adictivas, Complejo Hospitalario Universitario de Ourense, España \\ **Centro de Investigación Biomédica en Red de Salud Mental (CIBERSAM), Oviedo, España
}

$\mathrm{L}$ as adicciones y la violencia interpersonal, fuertemente vinculadas entre sí, son un reto para la salud pública. Obviamente, las adicciones pueden incrementar el riesgo de violencia interpersonal directamente a través de la intoxicación, la financiación del consumo de drogas o por formar parte de un mercado de drogas, ilegal, y por tanto, violento. Sin embargo, también se han definido como significativos los siguientes factores, compartidos a nivel individual, para ambos problemas (Yu et al., 2012): padecer un trastorno internalizante, padecer un trastorno mental grave, ser varón, ser joven, tener una educación y un rendimiento escolar deficientes, ser agresivo y tener alteraciones de la personalidad que incluyan la impulsividad, la búsqueda de sensaciones y la falta de control ejecutivo.

Los síntomas internalizantes, la agresión, la impulsividad, la búsqueda de sensaciones y la falta de control ejecutivo son síntomas comunes de los Trastornos de Personalidad (TP) (Skodol et al., 2005); un grupo heterogéneo de trastornos con una prevalencia en población general que oscila entre un 4 y un 13\% (Yu et al., 2012). Por ello, no es sorprendente que se haya encontrado una clara asociación entre los TP y la violencia (Yu et al., 2012), con una Odds Ratio (OR) de 3,0 (intervalo de confianza (IC:): 2,6 a 3,5). Se ha reporta- do una asociación mayor para el Trastorno Antisocial de la Personalidad (TAP), con una OR de 12,8 (IC: 7,4 a 14) (Yu et al., 2012), un riesgo similar al que presentan los adictos al alcohol o a las drogas. Así que, para evitar un acto violento, hay que detener a siete sujetos con TAP (Yu et al., 2012). Los individuos con TAP también tienen un mayor riesgo de reincidencia (Yu et al., 2012).

El TAP parece ser importante para comprender la violencia interpersonal e intentar prevenirla, pero,¿qué es el TAP? Para el DSM es «un patrón generalizado de desprecio hacia y violación de los derechos de los demás que se inicia en la niñez o en la adolescencia temprana y continúa en la edad adulta" (Lynam y Vachon, 2012). Así que el TAP trata de ser antisocial, y ser antisocial es lo que hace que una persona reciba un diagnóstico de TAP. Esto no es muy útil. Es lo mismo que decir que alguien está enfermo porque tiene fiebre, y que tiene fiebre porque está enfermo. Es cierto, pero este reduccionismo no nos lleva a ninguna parte en la búsqueda de soluciones. Necesitamos una aproximación más específica. El DSM establece que se requieren tres o más de los siguientes signos y síntomas para confirmar un diagnóstico de TAP: no seguir las normas sociales, indiferencia temeraria hacia la propia seguridad o la de los demás, irresponsabilidad persistente, deshonestidad, impulsividad,

Recibido: Febrero 2016; Aceptado: Marzo 2016

Enviar correspondencia a:

Unidad de Conductas Adictivas, Hospital Santa María Nai, Complejo Hospitalario Universitario de Ourense, Ramón Puga 52-56, 32005, Ourense E-mail: gerardo.florez.menendez@sergas.es. 
irritabilidad y agresividad y falta de remordimientos. Los primeros cuatro son los signos necesarias para afirmar que un individuo muestra "un patrón generalizado de desprecio hacia y violación de los derechos de los demás...; los tres últimos son necesarios para clasificar a un individuo dentro de ese patrón. Como hemos mencionado anteriormente, la impulsividad, la irritabilidad y la agresividad también son riesgos compartidos por las adicciones y la violencia interpersonal. Pero, ¿qué hay de la falta de remordimientos?

La falta de remordimientos se ha descrito como uno de los síntomas centrales que los clínicos e investigadores deben tener en cuenta cuando intentan detectar la psicopatía, otro tipo de TP cercano, y que generalmente se confunde, con el TAP y la conducta antisocial (Cooke y Michie, 2001). Esta confusión es absolutamente comprensible, ya que la psicopatía no se incluye en los manuales de diagnóstico como el DSM o la CIE, y, aún más importante, porque ambos trastornos comparten algunas características.

La psicopatía se ha descrito como un patrón de: (1) insensibilidad y ausencia de emociones (callous and unemotional (CU)), afectos que reflejan una deficiente experiencia emocional, (2) un estilo interpersonal grandioso y arrogante, y (3) una conducta impulsiva generalizada (Cooke y Michie, 2001; Hare et al., 2000). Si comparamos ambos patrones, podemos establecer fácilmente que la impulsividad es una característica común de ambos trastornos. Esta característica común hace que ambos trastornos formen parte del síndrome externalizante, e incluso explica por qué existe un solapamiento genético entre ambos (Larsson et al., 2007). Por lo tanto, la impulsividad es la clave para definir que una persona tiene un trastorno externalizante, pero no todos los externalizantes presentan conducta antisocial, o TAP, o psicopatía. No, la impulsividad es el terreno común, necesario, pero no suficiente. Se requiere al menos uno de otros dos factores (Larsson et al., 2007): un ambiente que promueva la conducta antisocial y/o ser CU, que claramente se relaciona con la falta de remordimiento. Antes de comentar estos factores, consideremos la naturaleza y las bases biológicas de la impulsividad.

En el primer párrafo, la impulsividad, la búsqueda de sensaciones y la falta de control ejecutivo se definieron como alteraciones de la personalidad que se relacionaban al mismo tiempo con las adicciones y la violencia interpersonal. Como en el caso del TAP y la psicopatía, también estos términos se confunden. La búsqueda de sensaciones o de la novedad es un constructo caracterizado por la búsqueda de nuevos estímulos incluso ante un mayor riesgo de sufrir daño $(\mathrm{Mu}-$ jica-Parodi et al., 2014). Las vías dopaminérgicas de los circuitos cerebrales motivacionales y de la acción modulan este comportamiento fuertemente biológico y hereditario. Esta conducta tiene sus beneficios y riesgos, especialmente si comparamos a las personas que puntúan alto en la búsqueda de la novedad-externalizadores, con una tendencia a buscar recompensas emocionales positivas- versus los que puntúan bajo en la búsqueda de la novedad- internalizadores, con una tendencia a evitar el castigo emocional negativo. Un desarrollo evolutivo correcto requiere un equilibrio entre los dos. Ninguno de ellos es a priori patológico en un individuo si existe flexibilidad conductual. En el caso de la búsqueda de la novedad, esta flexibilidad se pierde cuando falta el control ejecutivo, y en este caso, aparece la impulsividad. Desde este punto de vista, es clara la distinción entre el "valiente", que siente miedo y lo supera organizando su conducta para reaccionar ante el peligro, el "cobarde", que siente miedo y huye porque piensa que no es capaz de manejar el peligro, y el "temerario", que no siente miedo, por lo que ignora el peligro (Mujica-Parodi et al., 2014).

Los buscadores de sensaciones no-patológicos tienen suficiente cerebro como para ser conscientes del riesgo, controlar el miedo que surge debido a ese riesgo, y organizar una conducta que les permita lograr su meta. Los buscadores de sensaciones patológicos, personas impulsivas, también podrían lograr la meta, pero carecen del cerebro para ver el peligro y sentir miedo, por lo que no organizan su conducta, simplemente persiguen la meta. Ahora, ¿qué circuitos cerebrales son necesarios para ser "valiente" y no "temerario"? Los que proporcionan control ejecutivo (Mujica-Parodi et al., 2014). Es importante recordar que aquí no estamos hablando de conducta antisocial, sino de conducta impulsiva, cuyas consecuencias pueden ser prosociales o antisociales. La investigación ha mostrado constantemente que las regiones Prefrontales del cerebro y la Amígdala son estructuras fundamentales para el control ejecutivo. La Amígdala, un área subcortical compleja que regula el aprendizaje condicionado no consciente basado en claves emocionales y fisiológicas, procesa automáticamente el miedo que se siente y envía la información a la Corteza ÓrbitoFrontal (COF) donde el miedo se relaciona con el peligro y, con la ayuda del resto de la corteza Prefrontal, se organiza una conducta estructurada para superar el miedo, controlar el peligro y alcanzar la meta (Mujica- Parodi et al., 2014).

La ira, con o sin miedo, es otra emoción que puede desencadenar la agresión impulsiva o reactiva. En este caso, una Amígdala hipersensible reacciona ante la ira que acompaña a la conducta agresiva automática, destinada a eliminar o suprimir los estímulos ambientales presentes que han sido automáticamente percibidos como la fuente de la amenaza o de la frustración. A las regiones Prefrontales, como la COF y la Corteza Cingulada Anterior (CCA), les falta control ejecutivo para inhibir la conducta agresiva (Rosell y Siever, 2015; Siever, 2008).

Estudios de neuroimagen han demostrado que el adelgazamiento de la corteza Prefrontal y una reducción en su funcionamiento se correlacionan con la impulsividad $(\mathrm{Mu}-$ jica-Parodi et al., 2014) y con los individuos antisociales (Yang y Raine, 2009). En un meta-análisis de 43 estudios, un incremento en la conducta antisocial se asoció con el deterioro estructural y funcional de la COF derecha (un área 
que ayuda a inhibir comportamientos no planeados que se consideran inadecuados para calibrar el comportamiento según las claves sociales, cuyos premios y castigos no son tan inmediatos como los de los comportamientos impulsados por emociones), de la CCA derecha (un área que ayuda a procesar las emociones para controlar los comportamientos provocadas por las emociones) y de la corteza Prefrontal DorsoLateral (CPDL) izquierda (un área que ayuda a alcanzar la flexibilidad cognitiva) (Yang y Raine, 2009). En definitiva, cuando la búsqueda de sensaciones se descarría debido a la falta de control ejecutivo, las personas se vuelven impulsivas y aumenta el riesgo de agresión impulsiva y de la violencia reactiva y no planificada, no importa con qué diagnóstico se haya etiquetado al individuo (Blair y Lee, 2013). El abuso de drogas y las adicciones son otra consecuencia de la impulsividad, y es por lo que la impulsividad es un factor de riesgo común para ambos problemas. Cuando una persona impulsiva abusa de las drogas o del alcohol, aumenta el riesgo de ser violento y agresivo de manera antisocial (Romero-Martínez y Moya-Albiol, 2015). Obviamente, las personas impulsivas pueden ser antisociales antes de abusar de sustancias adictivas, si el ambiente familiar o social donde se criaron promueve la conducta antisocial, ya sea debido a la privación socioeconómica y/o una forma criminal de vida; o si presentan rasgos CU y carecen de remordimientos.

Como hemos mencionado anteriormente, ser insensible y carente de emociones (CU) es una de las tres dimensiones de la psicopatía. ¿De dónde viene esta dimensión? De nuevo, se invoca a la Amígdala y a la corteza Prefrontal, pero de una forma diferente. La Amígdala no sólo se implica en el procesamiento de las emociones internas, sino también de las expresiones emocionales de los demás, especialmente el miedo, el dolor y la tristeza, signos de malestar (Blair, 2008). Como es un área de aprendizaje automático en función del valor asignado a los estímulos, es fácil comprender que las expresiones emocionales de los demás también se van a utilizar para aprender asociaciones de amenaza/castigo y de recompensa (Blair, 2008). La Corteza Prefrontal VentroMedial (CPVM) es otra área Prefrontral de control ejecutivo, relacionada con la regulación emocional a través de la representación del valor emocional de la información (Blair, 2008). Los niños psicopáticos-lo cual significa que puntúan alto en rasgos CU-muestran alteraciones en la atención emocional similares a los de pacientes con lesiones en la Amígdala (Blair, 2008), con una disminución en la respuesta a las expresiones de miedo, dolor y tristeza (expresiones faciales, tonos de voz y gestos corporales) y un deterioro en el aprendizaje por refuerzo condicionado asociado a estímulos provocados por dichas expresiones. Cuando no se recibe la información emocional correcta de refuerzo de la Amígdala, la conectividad funcional entre las dos áreas es más débil (Herpers et al., 2012), y la CPVM no puede representarla para una correcta toma de decisiones. Los niños psicópaticos carecen de una habilidad básica para la socialización, algo que se puede aprender, pero no enseñar, ya que es aprendizaje condicionado. Básicamente, que el miedo, el dolor y la tristeza en los que los rodean son aversivos y tienen que ser evitados al igual que otros estímulos negativos. Sin esta información emocional crucial, la CPVM no puede ayudar a otras áreas Prefrontales en la correcta toma de decisiones sociales. Los niños y adultos psicopáticos no muestran empatía emocional, pero tienen empatía cognitiva, saben lo que el miedo, el dolor y la tristeza significan y lo que está bien y mal (Blair et al., 2006). Pero, cuando el miedo, el dolor y la tristeza aparecen a su alrededor, no sienten malestar, por lo que no sienten ningún impulso emocional de ayudar a los demás a superar su miedo, dolor y tristeza, no tienen comportamientos empáticos y siguen intentando lograr su objetivo (Blair, 2008; Blair y Mitchell, 2009). Esta ceguera emocional puede ser considerada como un déficit atencional (Blair y Mitchell, 2009; Newman et al., 2010), se ignora la información emocional relevante de abajo-arriba (bottom-up) para una adecuada socialización y debido a esto, la correcta toma de decisiones morales y sociales se ve afectada (Blair et al., 2006); los psicópatas sólo consideran la información de arriba-abajo (top-down) que les impulsa hacia el logro de sus objetivos (Blair y Mitchell, 2009). En definitiva, los psicópatas ignoran las normas basadas en la compasión y la justicia (Blair et al., 2013). De esta manera, el problema comienza como un déficit emocional, o un priming afectivo más débil, que interfiere con el juicio moral (Young y Koenigs, 2007), de modo que estas personas son vistas por los demás como CU, sin empatía ni vínculos, faltos de culpa y remordimientos, mostrando emociones poco profundas y relaciones interpersonales superficiales. Por esta razón, la psicopatía es el único trastorno mental en el que aumenta el riesgo de agresión instrumental; este tipo de violencia tiene un propósito y se orienta a un objetivo y puede ser planeada y ejecutada sin activación autonómica. Los individuos psicopáticos carecen de todos los frenos emocionales y morales que impiden a la gente normal realizar la violencia instrumental que les viene a la mente. Esto no significa que el riesgo de agresión reactiva no aumente en los psicópatas, realmente este riesgo está aumentado, también pueden ser impulsivos, así como "temerarios", o sentirse enfadados y frustrados (Blair, 2008). Otra cuestión que ha de tenerse en cuenta en relación con la violencia y la psicopatía es que no sólo muestran una respuesta emocional reducida hacia las expresiones de miedo, dolor y tristeza en los demás, sino también a las expresiones de amenaza (Blair et al., 2013). En situaciones de amenaza interpersonal, estas personas muestran una menor respuesta del Cortisol, altos niveles de Testosterona, y menos activación fisiológica (Herpers et al., 2012). De nuevo, se ve afectada su toma de decisiones basada en el refuerzo y muestran menos malestar ante el valor emocional negativo de ser herido por los demás, lo que los vuelve más violentos y agresivos. 
Las investigaciones del rasgo $\mathrm{CU}$ han revelado lo siguiente: el rasgo es más dimensional que categórico (Blair y Lee, 2013), como ocurre con la búsqueda de la novedad, los niveles flexibles de contagio empático son útiles desde una perspectiva evolutiva, especialmente para los varones, que tienen más comportamientos dominantes evolutivos y podrían necesitar ser agresivos y violentos en más ocasiones a lo largo de su vida comparados con las mujeres; el rasgo CU puede ser evaluado con fiabilidad a partir de la infancia (Herpers et al., 2012); los niños [de ambos sexos] afectados son generalmente más agresivos (Herpers et al., 2012); los factores genéticos contribuyen de forma importante a su expresión (el 40-78\% de la varianza del rasgo es atribuible a influencias genéticas), mientras que la conducta antisocial parece ser más impulsada por el ambiente (Herpers et al., 2012; Larsson et al., 2006; Larsson et al., 2007; Viding y McCrory, 2012); a diferencia de la conducta antisocial, el rasgo CU se vuelve más estable con la edad y se asocia con más problemas de conducta y comportamientos violentos (Herpers et al., 2012; Herpers et al., 2014; Lynam et al., 2007), de tal forma que este rasgo puede ser considerado una tendencia básica, mientras que la conducta antisocial es más una adaptación a factores ambientales (Cooke y Michie, 2001).

Una importante observación ha de hacerse acerca de la relación de tener un rasgo $\mathrm{CU}$, recibir un diagnóstico de psicopatía y ser antisocial. Como la psicopatía es un diagnóstico usado en los contextos forenses que tienen una relación directa con el sistema de justicia penal, la mayoría, si no todos los adultos etiquetados como psicópatas, mostrarán una combinación de los rasgos de conducta antisocial y CU. Necesitan presentar rasgos CU para cumplir los criterios de psicopatía, pero también tener conducta antisocial para ser evaluados por un forense que realice el diagnóstico (Viding y McCrory, 2012). ¿Significa esto que los rasgos CU siempre se acompañan de conducta antisocial? No necesariamente (Benning et al., 2003). Podemos encontrar individuos con rasgos CU que no son impulsivos y no toman o abusan de las drogas, y que han un encontrado un nicho ecológico-social donde pueden plasmar su conducta CU sin ser entrevistados por personal forense (por ejemplo, el usurero Sr. Scrooge de la novela Un cuento de Navidad de Dickens). Las investigaciones con niños también muestran que tienen rasgos CU antes de ser etiquetados como antisociales (Viding y McCrory, 2012). De esta manera, la impulsividad y el abuso de drogas hacen que las personas con rasgos CU muestren más conducta antisocial que los llevará a cometer delitos, generalmente con más violencia que otros delincuentes, y eso los arrojará a una evaluación forense donde recibirán la etiqueta de psicópata.

Como se señaló anteriormente, la falta de remordimientos, un síntoma de TAP y el rasgo CU- una dimensión psicopática-pueden interpretarse como la misma cosa. Así, podemos establecer que alguien que presenta un rasgo CU seguramente carece de remordimientos. Pero no todas las personas que puntúan alto en la falta de remordimientos tienen un rasgo CU y deben considerarse psicópatas. Se requiere una buena evaluación para averiguar si alguien muestra falta de remordimientos porque él o ella no siente empatía emocional, algo claramente psicopático, o si es algo determinado por el medio ambiente, y el individuo puede mostrar empatía emocional hacia alguien, lo cual sería más bien antisocial (Cooke y Michie, 2001; Hare et al., 2000; Sellbom et al., 2015).

¿Qué relación han encontrado las investigaciones entre la psicopatía y el uso de sustancias? La investigación en la que se ha utilizado el Psychopathic Checklist List Revised (PCL-R) ,una herramienta forense para el diagnóstico de psicopatía (Hare et al., 2000), ha permitido responder a esta pregunta. El PCL-R puede dividirse en dos factores: (1) interpersonal y afectivo e (2) impulsivo y estilo de vida antisocial (Hare et al., 2000; Storey et al., 2015). Estudios bien replicados informan que la adicción al alcohol y las drogas y las puntuaciones totales en el PCL- R presentan una correlación de moderada a baja (Hemphill et al., 1994), siendo más fuerte para la adicción a drogas no alcohólicas. Pero también muestran que el Factor 2 correlaciona más fuertemente con la adicción a drogas y alcohol que el Factor 1 (Hemphill et al., 1994). Esta correlación también está presente cuando se consideran otras variables como el número de sustancias probadas, la edad de la primera intoxicación alcohólica y el número de cargos o condenas por delitos relacionados con las drogas (Hemphill et al., 1994). Por lo tanto, el uso y abuso de sustancias en personas psicopáticas está más relacionado con ser "temerario" que con ser CU, que también es un factor común para la violencia interpersonal. Esta diferencia, como algunas investigaciones sugieren, puede ser más intensa en mujeres psicopáticas, donde el Factor 1 incluso podría ser un factor protector contra el abuso de sustancias (Schulz et al., 2015).

Como conclusión, se ofrece para los clínicos que trabajen en Unidades de Tratamiento de las Adicciones una lista de factores de riesgo a considerar a la hora de evaluar el riesgo de violencia interpersonal (ver Tabla 1).

No todos estos signos y síntomas predicen el mismo nivel de riesgo, pero podemos asumir que cuantos más muestre un paciente, más alto es el riesgo. Sólo él último punto se relaciona claramente con la violencia instrumental, aunque la financiación del consumo de alcohol y drogas también podría estar relacionada. El resto de los puntos se asocian con la violencia reactiva. Por lo tanto, si un joven varón intoxicado, con una historia previa de comportamientos impulsivos y condenas penales por violencia, entra en tu oficina y, muy irritado, exige que le financies su consumo de alcohol y drogas, probablemente deberás hacer algo para garantizar tu seguridad personal, especialmente si sabes que este paciente específico tiene un rasgo CU estable.

Los clínicos interesados en adquirir más conocimientos de estos temas deberían intentar recibir entrenamiento 
Tabla 1. Lista de factores de riesgo a considerar para la evaluación del riesgo de violencia interpersonal en Unidades de Tratamiento de las Adicciones.

\footnotetext{
- El paciente es joven.

- El paciente es varón.

- El paciente muestra un nivel de educación completada bajo.

- El paciente es un buscador de sensaciones.

- El paciente padece un trastorno de salud mental.

- El paciente padece un trastorno de personalidad.

- El paciente es “temerario”, impulsivo y carece de control ejecutivo, esté intoxicado o no; la detección de este comportamiento cuando no existe intoxicación es más relevante.

- El paciente se irrita, enoja o frustra fácilmente, esté intoxicado o no; la detección de este comportamiento cuando no existe intoxicación es más relevante.

- El paciente proviene de y vive en un ambiente criminal.

- El paciente financia el consumo de alcohol o de drogas mediante acciones violentas.

- El paciente utiliza sustancias que incrementan la impulsividad y la irritabilidad, tales como el alcohol o la cocaína, hasta el punto de intoxicarse.

- El paciente muestra un rasgo de insensibilidad y carencia de emociones estable.
}

en la evaluación de la psicopatía, con herramientas como la Comprehensive Assessment of Psychopathic Personality (CAPP) o el PCL-R, y en la evaluación de riesgo, con herramientas como el Historical Clinical Risk Management (HCR-20 V3).

\section{Conflicto de intereses}

El autor declara la inexistencia de conflictos de intereses.

\section{Referencias}

Benning, S.D., Patrick, C.J., Hicks, B.M., Blonigen, D.M. y Krueger, R.F. (2003). Factor structure of the psychopathic personality inventory: validity and implications for clinical assessment. Psychological Assessment, 15, 340-350. doi:10.1037/1040-3590.15.3.340

Blair, K.S., Richell, R.A., Mitchell, D.G., Leonard, A., Morton, J. y Blair, R.J. (2006). They know the words, but not the music: affective and semantic priming in individuals with psychopathy. Biological Psychology, 73, 114-123. doi:10.1016/j.biopsycho.2005.12.006

Blair, R.J. (2008). The amygdala and ventromedial prefrontal cortex: functional contributions and dysfunction in psychopathy. Philosophical Transactions of the Royal Society of London. Series B: Biological Sciences, 363, 2557-2565. doi:10.1098/rstb.2008.0027

Blair, R.J. y Lee, T.M. (2013). The social cognitive neuroscience of aggression, violence, and psychopathy. Social
Neuroscience, 8, 108-111. doi:10.1080/17470919.2012.75 7869

Blair, R.J. y Mitchell, D.G. (2009). Psychopathy, attention and emotion. Psychological Medicine, 39, 543-555. doi:10.1017/S0033291708003991

Blair, R.J., White, S.F., Meffert, H. y Hwang, S. (2013). Emotional learning and the development of differential moralities: implications from research on psychopathy. Annals of the New York Academy of Sciences, 1299, 36-41. doi:10.1111/nyas.12169

Cooke, D.J. y Michie, C. (2001). Refining the construct of psychopathy: towards a hierarchical model. Psychological Assessment, 13, 171-188.

Hare, R.D., Clark, D., Grann, M. y Thornton, D. (2000). Psychopathy and the predictive validity of the PCL-R: an international perspective. Behavioral Sciences and the Law, 18, 623-645.

Hemphill, J.F., Hart, S.D. y D. Hare, R. (1994). Psychopathy and Substance Use. Journal of Personality Disorders, 8, 169180. doi:10.1521/pedi.1994.8.3.169

Herpers, P.C., Rommelse, N.N., Bons, D.M., Buitelaar, J.K. y Scheepers, F.E. (2012). Callous-unemotional traits as a cross-disorders construct. Social Psychiatry and Psychiatric Epidemiology, 47, 2045-2064. doi:10.1007/s00127-012-0513-x

Herpers, P.C., Scheepers, F.E., Bons, D.M., Buitelaar, J.K. y Rommelse, N.N. (2014). The cognitive and neural correlates of psychopathy and especially callous-unemotional traits in youths: a systematic review of the evidence. $D e-$ velopment and Psychopathology, 26, 245-273. doi:10.1017/ S0954579413000527

Larsson, H., Andershed, H. y Lichtenstein, P. (2006). A genetic factor explains most of the variation in the psychopathic personality. Journal of Abnormal Psychology, 115, 221-230. doi:10.1037/0021-843X.115.2.221

Larsson, H., Tuvblad, C., Rijsdijk, F.V., Andershed, H., Grann, M. y Lichtenstein, P. (2007). A common genetic factor explains the association between psychopathic personality and antisocial behavior. Psychological Medicine, 37, 15-26. doi:10.1017/S003329170600907X

Lynam, D.R., Caspi, A., Moffitt, T.E., Loeber, R. y Stouthamer-Loeber, M. (2007). Longitudinal evidence that psychopathy scores in early adolescence predict adult psychopathy. Journal of Abnormal Psychology, 116, 155-165. doi:10.1037/0021-843X.116.1.155

Lynam, D.R. y Vachon, D.D. (2012). Antisocial personality disorder in DSM-5: missteps and missed opportunities. Personality Disorders, 3, 483-495. doi:10.1037/per0000006

Mujica-Parodi, L.R., Carlson, J.M., Cha, J. y Rubin, D. (2014). The fine line between 'brave' and 'reckless': amygdala reactivity and regulation predict recognition of risk. Neuroimage, 103, 1-9. doi:10.1016/j.neuroimage.2014.08.038

Newman, J.P., Curtin, J.J., Bertsch, J.D. y Baskin-Sommers, A.R. (2010). Attention moderates the fearlessness of 
psychopathic offenders. Biological Psychiatry, 67, 66-70. doi:10.1016/j.biopsych.2009.07.035

Romero-Martínez, A. y Moya-Albiol, L. (2015). Neuropsychological impairments associated with the relation between cocaine abuse and violence: neurological facilitation mechanisms. Adicciones, 27, 64-74.

Rosell, D.R. y Siever, L.J. (2015). The neurobiology of aggression and violence. CNS Spectrums, 20, 254-279. doi:10.1017/s109285291500019x

Schulz, N., Murphy, B. y Verona, E. (2015). Gender Differences in Psychopathy Links to Drug Use. Law and Human Behavior, [Epub ahead of print]. doi:10.1037/ lhb0000165

Sellbom, M., Cooke, D.J. y Hart, S.D. (2015). Construct Validity of the Comprehensive Assessment of Psychopathic Personality (CAPP) Concept Map: Getting Closer to the Core of Psychopathy. International Journal of Forensic Mental Health, 14 , 172-180. doi:10.1080/14999013.2015.10 85112

Siever, L.J. (2008). Neurobiology of aggression and violence. American Journal of Psychiatry 165, 429-442. doi:10.1176/appi.ajp.2008.07111774

Skodol, A.E., Gunderson, J.G., Shea, M.T., McGlashan, T.H., Morey, L.C., Sanislow, C.A.,... Stout, R.L. (2005). The Collaborative Longitudinal Personality Disorders Study (CLPS): overview and implications. Journal of Personality Disorders, 19, 487-504. doi:10.1521/pedi.2005.19.5.487

Storey, J.E., Hart, S.D., Cooke, D.J. y Michie, C. (2015). Psychometric Properties of the Hare Psychopathy Checklist-Revised (PCL-R) in a Representative Sample of Canadian Federal Offenders. Law and Human Behavior, [Epub ahead of print]. doi:10.1037/lhb0000174

Viding, E. y McCrory, E.J. (2012). Genetic and neurocognitive contributions to the development of psychopathy. Development and Psychopathology, 24, 969-983. doi:10.1017/S095457941200048X

Yang, Y. y Raine, A. (2009). Prefrontal structural and functional brain imaging findings in antisocial, violent, and psychopathic individuals: a meta-analysis. Psychiatry Research, 174, 81-88. doi:10.1016/j.pscychresns.2009.03.012

Young, L. y Koenigs, M. (2007). Investigating emotion in moral cognition: a review of evidence from functional neuroimaging and neuropsychology. British Medical Bulletin, 84, 69-79. doi:10.1093/bmb/ldm031

Yu, R., Geddes, J.R. y Fazel, S. (2012). Personality disorders, violence, and antisocial behavior: a systematic review and meta-regression analysis. Journal of Personality Disorders, 26, 775-792. doi:10.1521/pedi.2012.26.5.775 